\title{
How Could Device Security Against Unauthorized Internal Chip Access be Married with Design For Testability?
}

\author{
Peter Jacob \\ Empa Swiss Federal Laboratories for Materials Testing and Research \\ Dept. 173 Electronics/ Metrology - Reliability Center \\ Ueberlandstr. 129, CH-8600 Duebendorf Switzerland \\ Tel. +41-44-823-4288, Fax +41-44-823-4054, e-Mail: peter.Jacob@empa.ch
}

\begin{abstract}
New devices with security-critical functions like in moneyor credit cards frequently have protective top metal layers in order to avoid unauthorized internal chip-access for testing or modifying the device. However, design/process debug and FA need such access to accelerate learning for production ramp-up and design debugging. The paper shows a design approach how to allow authorized access for internal probing in such case.
\end{abstract}

\section{INTRODUCTION AND MOTIVATION}

In the recent years, security requirements for smart card chips significantly increased. This means not only protection against electrical access and -manipulation but also includes more and more limited or forbidden FIB accessibility in order to avoid hardware circuit changes and manipulations or unauthorized memory readouts. On the other hand, such design philosophy makes the ramp-up of new devices rather difficult and time-consuming. In some cases, FIB-made chip modification requests for design debugging became nearly impossible or can be accessed exclusively from the backside [1].

Both design debugging and failure analysis needs internal probing for failure diagnosis \& localisation purpose. State-of-the-art devices use protective layers, for example meander structures in the top metal, sometimes including metals underneath, covering at least all critical device areas. If this layer is locally interrupted (e.g. by FIB modifications), the device either becomes non-functional or deletes the memory content or even destroys itself when it is set under current next time. As a detection criterion for such meander interrupt, not only the ohmic resistance is used but also additional criteria like its (undistorted) capacitance or inductance values can be used to enhance the protection level. In such case, the detection needs an RLC evaluation circuitry. Thus, even if such meander should be repaired by FIB after an illegal access, the device would recognize the access attempt and become non-functional or even destroyed after being connected back to operational conditions. In the following chapters, the actual security concepts are explained and approaches how to marry them with the needs of modern failure analysis and debugging - without loosing their protective functions - are shown.

\section{PROTECTION STRATEGIES}

We analysed several chips bought from the market in order to study such protections in the frame of competitive analysis (however, these studies themselves are no subject of publication in this paper); in addition, some literature studies have been done. From these inputs we concluded towards our proposal. Embedding electrically inactive filler structures at least into the few top metal layers performed the first protection approaches (besides their major function for thermomechanical relaxation), as shown in fig 1. Some chip manufacturers prefer full metal layer coverage of the device. However, in case of thermal cycling and large, unstructured areas, such approach might

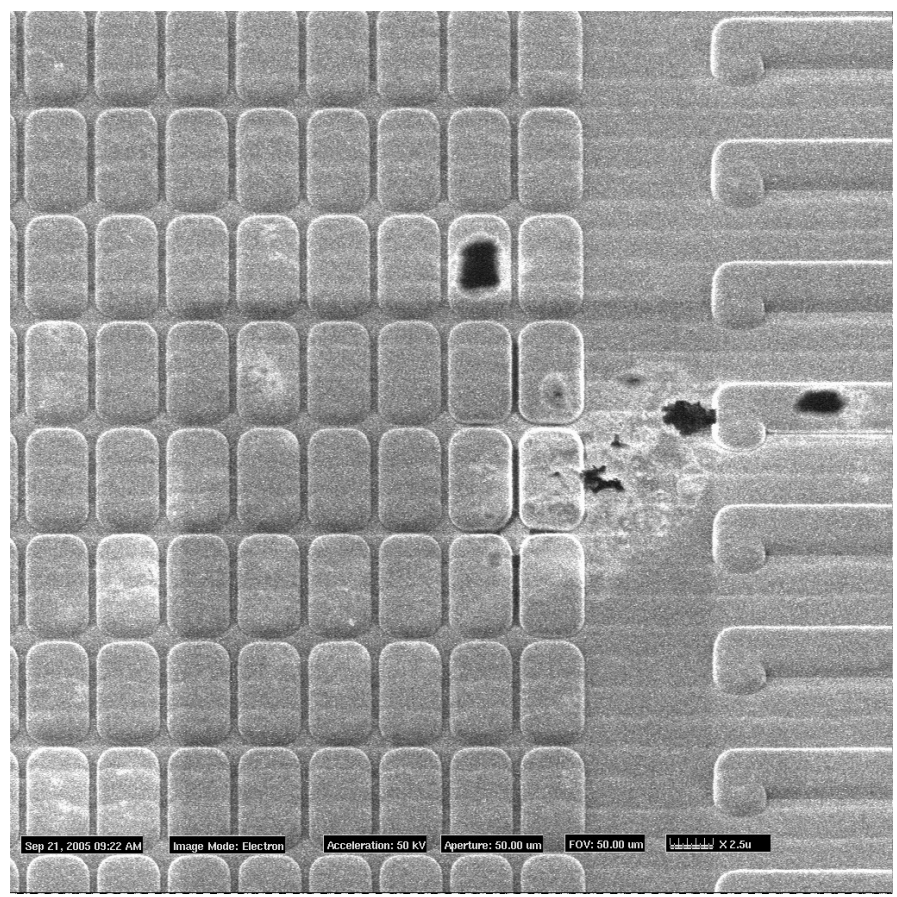

Fig.1: Fillers serve not only to reduce thermomechanical stress but also to hide structures 
become critical due to thermal expansion coefficient differences. Alternatively or in addition, access-critical structures can be placed underneath of thick top metal bus lines or underneath of pads. However, the security protection effect is relatively weak, since locally, these fillers or top metal lines could be removed respectively bypassed by means of FIB and thereafter, regular device modifications or internal probing could start. In a certain manner, these fillers prevent frontside access for optical analysis/ modification methods like emission microscopy, OBIRCH analysis or laser-controlled modifications, but, since many methods operate within the infrared range of light, they could be used anyway from the backside - which, however, needs substantial chemical and physical preparation efforts on the chip to be investigated in most of the cases [2].

More sophisticated approaches were selected by several manufacturers when using meander top metal line structures to protect the device surface against FIB access, as the example of fig. 2 shows. If the meander has been interrupted for instance by

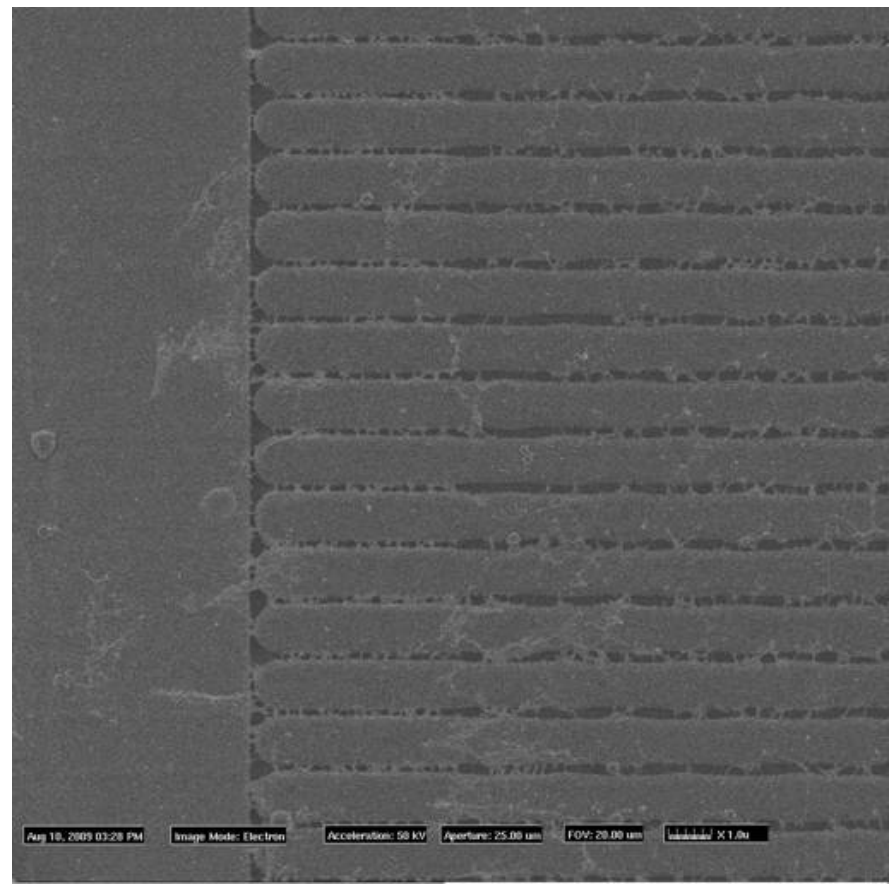

Fig.2: top view of a meander metal protector. In the metal layer underneath, the top metal lines are connected to a meander line

FIB in order to study the chip, the resistance change was self-recognized by the chip electronics and, as a consequence, the chip was either electrically blocked or it even destroyed itself electrically at the next electrical operation. A very effective protection approach is shown - as an example - in fig. 3: Four partial resistors R1-R4 are connected to a high-ohmic Wheatstone bridge. A comparator detects the equilibrium of this bridge. If - for example by an unauthorized FIB modification one of the resistors has been interrupted or changed its value, the

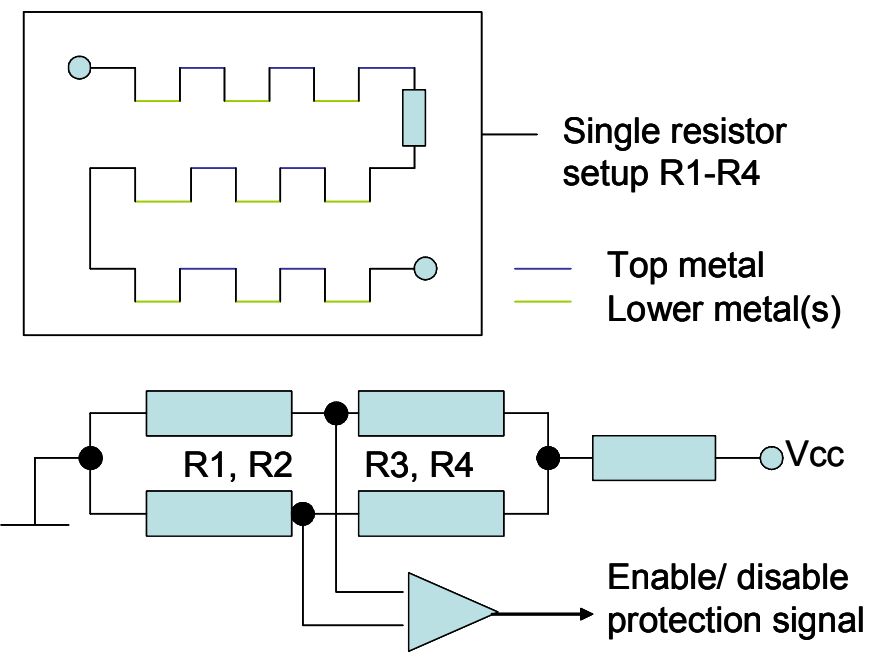

Fig. 3: chip-coating metal lines, as shown in fig. 2, can also be combined to a "Wheatstone" bridge circuitry, which can be adjusted very sensitive to detect external modifications

comparator will "see" the deranged equilibrium and send a "protection alarm" signal, which either destroys the circuit or disables its functionality.

A furthermore improved development of such protection included also capacitance or even inductance change sensitivity of the shielding layer. Fig. 4 shows the scheme/ function principle of such protection. In both cases of figures 3 and 4, filler structures or parts of them can be included as via chain structures in the circuitry and, thus, become a part of the electrical protection circuitry.

In some cases, only the memory is protected against unauthorized access or readout. Frequently mathematical encryptions/ codes are used for protection instead of or in addition to hardware solutions.

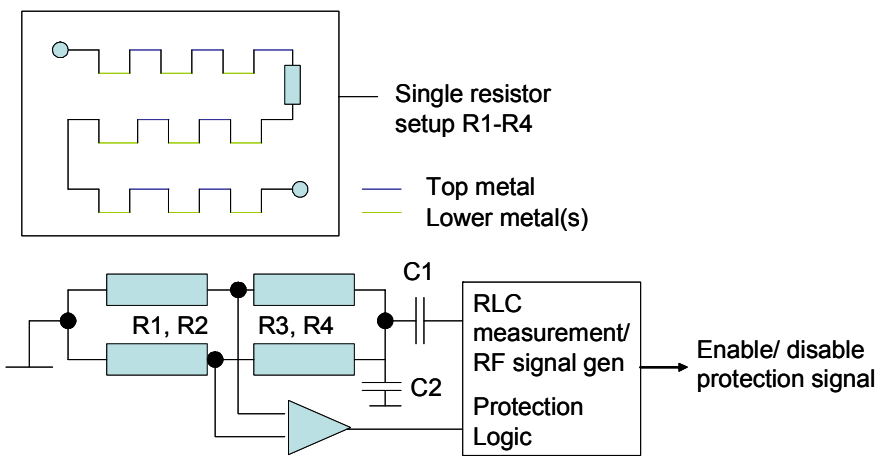

Fig. 4: A further sophistication of fig. 3 circuitry includes the detection of the capacitance versus sub-metal layers and/ or the circuitry inductance. Thus, the impedance becomes detectable, even in those cases, where FIB-accessing openings of the meander structures had been repaired after the related device modification. However, such approach needs RLC detecting and, due to its complexity, it might become an inertial source of yield failures 
A specific protection against FIB-access has been described in [3]: The dielectric material between metal lines is made in a manner that it becomes conductive under FIB ion bombardment, thus short-circuiting all metal lines within the FIB modification window. Using this approach, the chip will destroy itself when FIB modification would be tried.

Since backside analysis methods and backside FIB access developed further in the recent years, the question came up how to protect the devices against backside access. In [4], such protection consists of an active backside antenna, which responds to the transmission signals of a frontside antenna. In case of hurting one of both antennas, which coat both chip front resp. backside, the transmission will be resonance-disturbed and a chip protection or -destruction circuitry can be activated.

\section{HOW TO MAINTAIN SECURITY AND TO MANAGE TESTING AND DEBUGGING AT THE SAME TIME}

\section{Testing and Debugging Requirements}

During the ramp-up phase, standard- and even extended test programs usually don't give enough information for evaluation of process windows, functional guard band definitions beyond specified operational data and engineering and debugging of the chip design. Either this layout already performs specific needle probe test pads, or, in many cases of design reviews/ improvements, specific circuitry must be made accessible by means of FIB-made probe pads according to designer's or test engineer's requests.

Above these basic requests, the device should allow the localisation of defects by the most common localisation methods emission microscopy and OBIRCH. In case of more than two metal layers and lots of filler structures, the device surface access to these methods becomes rather difficult; on the other hand, these methods have been further developed significantly in the recent years and the backside access including backlapping preparation procedures meanwhile became most common within the failure analysis community $[5,6]$. The only limitations are given by highly doped bulk silicon or SOI's (Silicon On Insulator), which may block or dissipate the backside access of infrared light.

\section{Security Requirements}

The security requirements focus in two different directions: one goal is to make reverse chip engineering by competitors as difficult as possible and the other goal is to prevent unauthorized access or modification of data stored within the device memories. Especially money- or sensitive access cards must be absolutely safe against such attacks.

Whilst the latter requirement can be reached rather successfully by using tricky mathematical algorithms, passwords etc., the fulfilling of the first task may become a boomerang for the own factory's high volume production yield ramp-up and sometimes even for the design debugging. Therefore this point needs to be discussed more deep and is the core of this paper.

\section{Useful solution approaches}

The proposed solution for the failure analyst's problem in case of process- or design-supporting analysis is, to perform several small test pads into the regular design of the layer underneath of the protective metal layer. To make it perfect, some or all of such pads can be masked as "regular" filler structures with hidden vias underneath for connecting them. One (or some more) of these pads in the top metal layer, disguised as fillers and only known to the designer and failure analyst, ends in a resistor/ capacitor network which exactly replaces the protection layer or bypasses it's protective function. This gives two advantages at the same time:

First, if a legal engineering access would be needed, the protective layer may be locally been FIB removed, if the "secret pad" is connected to a dedicated reference point by FIB, thus replacing, deactivating or bypassing the protective layer. The second advantage is to have most of the important test pads within the second metal layer from top or even in the top layer. FIB-access to make test pads for internal needle probing becomes more easy and reliable. Since numerous test pads should be designed into the $2^{\text {nd }}$ metal layer from top, the secret "protective layer inactivation" pad could be easily hidden among other (access or dummy) pads or even fillers. Top metal dummy pads, however, should never be just floating, since this would become obvious by (locally depassivated) FIB inspection due to passive potential contrast.

If the protective meander is scaled additionally, it can also be used for easy navigation towards test pads underneath, or, specific filler "squares" could be used directly as test pads for internal needle probing.

The FIB work to get access to test pads (or even to get access to the circuitry underneath) would work as following:

Step1: Interrupt the protective layer at the position of the "protective layer disable" pad(s) within the metal level underneath.

Step 2: Open the access to this pad and connect it to the assigned second "protection disable" pad as prescribed by the chip designer. With this connection, the circuitry for testing, whether the protective structure has been hurt, will be inactivated.

Step 3: Locally remove the protection layer above those test pads to which you need access.

Step 4: Open the passivation/ ILD in order to get electrical access to those test pads you need for failure analysis (or do device editing modifications as demanded by the chip designer).

After having finished all test pad access or chip modifications, the chip can be operated normally and the F/A measurements can be done normally without the chip would start to destroy itself.

The same approach could be applied in case of backside antenna access protection as described before.

In case of the use of dielectrics, which start to become conductive in case of (Ga-) ion bombardment from FIB, no solution can be given in order to maintain the isolation characteristics. In the future, the question might come up, whether such dielectric would change its behaviour, too, when using other FIB ion species, like e.g. Helium or Xenon or UV-laser ablation. Since such kind of equipment is very new on 
the market and expensive, the author knows no investigations on that issue, yet.

A completely other approach would be the use of laser- or electrically trimmed fuses (at least three should be considered for this purpose). A dedicated combination sequence of blown/ unblown fuses would allow activating or inactivating the chip-internal self-protection- or destruction-circuitry. However, such approach should be used only in case the related fuses could be hidden within a complete array of further fuses for other, regularly purposes of the specific device, for example memory redundancy fuses, configuration fuses etc as shown in fig. 5 .

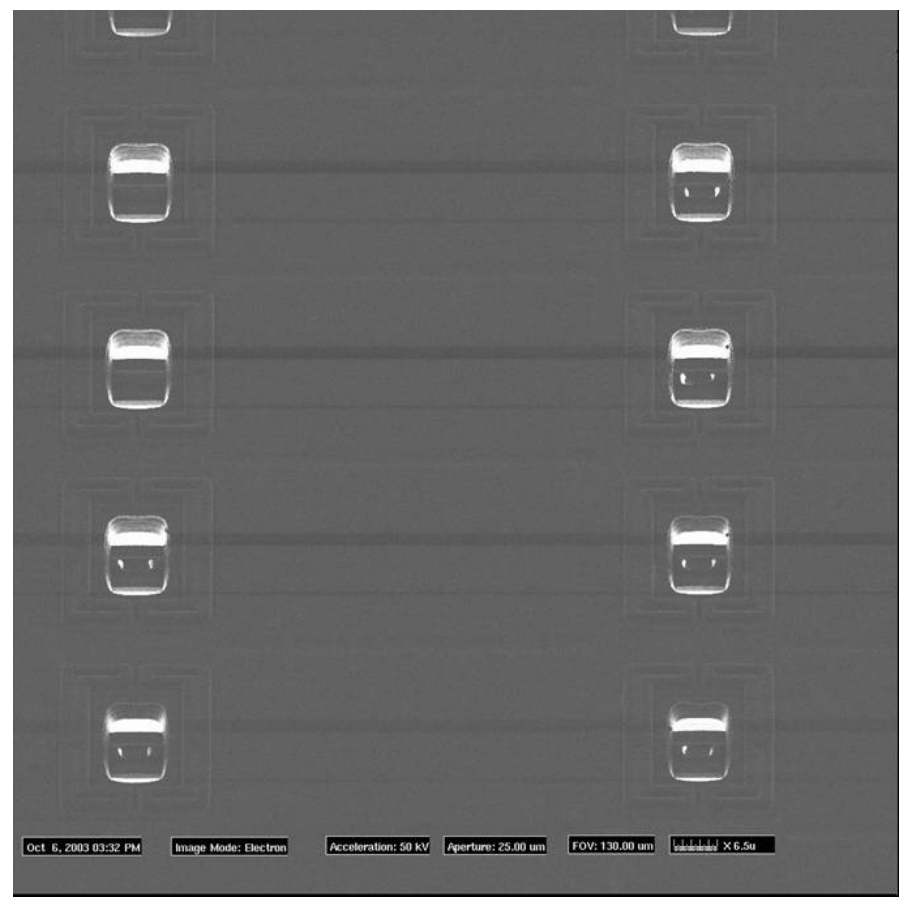

Fig. 5: fuse arrays are frequently used for customer- specific chip configuration, resonance adjustments or switching-on redundant circuitry. Within such fuse arrays, also fuses can be hidden, which enable or disable chip-protection circuitry

\section{OUTLOOK}

In the past years, the micro- and nanoscale manufacturing has been extended from chip towards small subsystems, like combinations of MEMS-sensors and semiconductor chips or OLEDs and semiconductor chips. Such combinations will allow extended concepts under inclusion of subsystems with respect to device security, too. For example, a sensor-chip combination could be "gated", so that only within few specific conditions or condition sequences, the device would be open for test mode access. However, such conditions must not disturb the device testability.

In total, such an approach would combine the requests of device security against illegal external manipulations with the needs of design debugging and legal failure analysis needs, too.

\section{CONCLUSION}

The paper has shown two approaches, how the protection against unauthorized device access and approved access and testability could be combined: One approach uses hidden fuse trimming to disable the protection circuitry; the second proposal is based on a FIB-based deactivating of the protection circuitry by FIB-connecting of two small top metal pads hidden in the top metal filler structures. These principles could also be combined each other as well as with dedicated algorithm commands to disable the protection circuitry. Such approach would both serve testability and supply sufficient security against unauthorised device access.

\section{REFERENCES}

[1] Rudolf Schlangen et al., „FIB backside circuit modification at the device level, allowing access to every circuit node with minimum impact on device performance by use of Atomic Force Probing", proc. of 33rd ISTFA conference, Nov. 2007, pp.34-40

[2] Christian Boit, Rudolf Schlangen, Uwe Kerst, Ted Lundquist, „Physical Techniques for Chip-Backside IC Debug in Nanotechnologies”, IEEE Design and Test of Computers, vol. 25 no.3, May 2008, pp. 250-257

[3] Franz Ungar, Gunter Schmid, "Semiconductor Chip with FIB Protection", US Patent 7038307, issued May02, 2006

[4] Itsik Mantin, John Walker, “Chip Attack Protection”, US patent publication number 20090001821, 2006, published 01.01.2009

[5] Jim Colvin, "BGA and Advanced Package Wire to Wire Bonding for Backside Emission Microscopy", proc. of ISTFA conference 1999, pp. 365-374

[6] Beaudoin, F. Imbert, G. Perdu, P. Trocque, C., "Current leakage fault localization using backside OBIRCH", proc. of 8th IPFA (International Symposium on the Physics and Failure Analysis of Integrated Circuits) conference 2001, pp.121-125 